\title{
Plasma Fatty Acid Composition Was Associated with Apelin Gene Expression in Human Adipose Tissues
}

\author{
Emad Yuzbashian $\left(\mathbb{D},{ }^{1}\right.$ Golaleh Asghari $\mathbb{D}^{(1,2}$, Nilofar Beheshti $\mathbb{D}^{\circ},{ }^{3}$ Mehdi Hedayati $(\mathbb{D})^{3}$ \\ Maryam Zarkesh $\left(\mathbb{D},{ }^{3}\right.$ Parvin Mirmiran $\mathbb{D}^{\mathbb{D}},{ }^{1,2}$ Afsoon Daneshafrooz $\mathbb{D}^{\mathrm{D}},{ }^{3}$ and Alireza Khalaj $\mathbb{D}^{4}$ \\ ${ }^{1}$ Nutrition and Endocrine Research Center, Research Institute for Endocrine Sciences, Shahid Beheshti University of \\ Medical Sciences, Tehran, Iran \\ ${ }^{2}$ Department of Clinical Nutrition and Dietetics, Faculty of Nutrition Sciences and Food Technology, National Nutrition and Food \\ Technology Research Institute, Shahid Beheshti University of Medical Sciences, Tehran, Iran \\ ${ }^{3}$ Cellular and Molecular Endocrine Research Center, Research Institute for Endocrine Sciences, Shahid Beheshti University of \\ Medical Sciences, Tehran, Iran \\ ${ }^{4}$ Tehran Obesity Treatment Center, Department of Surgery, Shahed University, Tehran, Iran
}

Correspondence should be addressed to Maryam Zarkesh; maryamzarkesh@yahoo.com and Parvin Mirmiran; mirmiran@endocrine.ac.ir

Received 22 September 2020; Accepted 13 September 2021; Published 6 October 2021

Academic Editor: Qiang Huo

Copyright (c) 2021 Emad Yuzbashian et al. This is an open access article distributed under the Creative Commons Attribution License, which permits unrestricted use, distribution, and reproduction in any medium, provided the original work is properly cited.

\begin{abstract}
Background. Apelin is an adipokine with an intermediatory role in obesity and insulin resistance, which can be modified by dietary intake. Aims. In this study, we aimed to determine the association of the plasma fatty acid composition with apelin plasma concentration and gene expression in visceral (VAT) and subcutaneous (SAT) adipose tissues. Methods. In this cross-sectional study, we recruited 179 patients aged 19-75 years who were candidates for elective surgery. Through the surgery, SAT and VAT were collected to measure apelin gene expression. Anthropometric measurements, fasting blood samples, and dietary intakes were collected before surgery. Free fatty acids (FFAs) in fasting whole plasma were measured using gas chromatography with flame ionization detection. Linear regression models were used to estimate standardized $\beta$ (STZ $\beta$ ) showing the association of individual and total FFAs with apelin gene expression after adjustment for potential confounding variables. Results. In multivariable analysis, we observed a significant positive association of total plasma free fatty acids (FFAs) (STZ $\beta=0.241, P=0.006$ ), saturated fatty acid (SFA) (STZ $\beta=0.336, P<0.001$ ), and monounsaturated fatty acid (MUFA) (STZ $\beta=0.313, P<0.001$ ) concentrations with apelin gene expression from VAT after controlling for age, sex, body mass index, homeostatic model assessment for insulin resistance (HOMA-IR), physical activity, and energy intake. In the SFA family, there was a direct association with plasma concentration of myristic acid (STZ $\beta=0.372, P<0.001$ ), pentadecanoic acid (STZ $\beta=0.252, P=0.002$ ), and heptadecanoic acid (STZ $\beta=0.407, P<0.001$ ) with apelin mRNA expression in VAT. There was no significant association between FFAs and apelin plasma concentration and SAT mRNA levels. Conclusions. In conclusion, circulating plasma FFAs, SFA, and MUFA had a positive association with apelin gene expression in VAT. It seems that plasma fatty acid composition may regulate apelin gene expression in VAT.
\end{abstract}

\section{Introduction}

Apelin is one of the adipokine family members, which is known as a highly conserved peptide and the endogenous ligand of APJ, a G-protein-coupled receptor [1,2]. Apelin expressed and secreted from a variety of tissues, including mainly from adipose tissue as well as the central nervous system and the gastrointestinal tract, the heart, the lungs, and with endocrine, paracrine, and autocrine function [1]. Apelin is involved in a wide range of physiological pathways, including energy homeostasis, regulation of body fluid, and immune, gastrointestinal, and cardiovascular functions [3]. 
Current animal studies indicated that apelin had an insulinsensitizing role and positively impacted glucose homeostasis [2]. The finding of a population-based study demonstrates that high apelin concentration was associated with reduced incidence of type 2 diabetes risk [4]; thus, it is implied that apelin may have a protective effect against metabolic disorders. Although some known physiological factors such as insulin and inflammation markers contribute to the regulation of apelin gene expression and plasma concentration, apelin regulation can be influenced by modifiable environmental factors $[5,6]$.

Free fatty acids (FFAs) have received remarkable interest in the context of metabolic disorders because they have potential roles in the inflammatory processes and gene expression of several organs $[7,8]$. A mediatory effect of circulating FFAs is proposed in the glucose-stimulated insulin production/release, decreasing glucose tolerance and insulin secretion $[4,9]$. Increased FFA concentration had an undesirable effect on insulin signaling [10]. Furthermore, observational and experimental studies indicated that the concentration of FFAs increases in obesity, which induces peripheral insulin resistance and decreased insulin secretion from pancreatic beta cells [11-14]. In addition to the impact of FFA concentration on glucose homeostasis, the evidence showed that adipokine concentration and gene expression could also be affected by the concentration of FFAs [15-17]. A direct association of erythrocyte membrane lauric and linoleic acids and an inverse association of total $\mathrm{n}-3$, eicosapentaenoic (EPA), and docosahexaenoic acids (DHA) with adiponectin and leptin concentrations were reported [15]. Besides, evidence illustrates that EPA and DHA reduced the relative gene expression of leptin, whereas palmitic or oleic acid had no significant effect [18].

In this regard, to the best of our knowledge, there is no study to examine the association of apelin gene expression from adipose tissues and specific FFAs. Given the importance of regulation of apelin on glucose homeostasis and the capability of FFAs to change adipose tissue metabolism, we aimed to investigate the association of circulating individual FFAs with apelin circulation and gene expression from visceral and subcutaneous human adipose tissue.

\section{Methods}

2.1. Participants. This crosssectional study was conducted on a convenience sampling method of 176 adults aged 19-75 years who were admitted for elective surgeries to the Mostafa Khomeini and Khatam Al-Anbia hospitals, Tehran, Iran. We included participants if they were admitted to the hospital less than three days. We excluded those with a chronic illness affecting diet such as diabetes or cancer, using any lipid-lowering or antiobesity or antidiabetic medication, or women with pregnancy and lactation. Approximately $100 \mathrm{mg}$ of subcutaneous adipose tissue (SAT) and visceral adipose tissue (VAT) samples were collected during their underlying surgeries in RNase- and DNase-free microtubes. Peripheral venous blood samples were also drawn after overnight fasting and were kept frozen at $-80^{\circ} \mathrm{C}$ until analysis.
Ethics approval was obtained from the ethics committee of the Research Institute for Endocrine Sciences of the Shahid Beheshti University of Medical Sciences (NO: IR.SBMU.ENDOCRINE.REC.1396.483), and we conducted the current study in accordance with the Declaration of Helsinki and RIES institutional guidelines. Written informed consent was obtained from all participants.

2.2. Plasma Free Fatty Acids. The measurement of plasma FFAs was described in detail elsewhere [19]. Briefly, fatty acid methyl esters were added to the gas chromatograph (Varian 450, City, USA) with flame ionization detection to quantify individual fatty acids. The device was equipped with a cyanopropyl siloxane 88 (CP-Sill 88) coated with siliconbased polymers (polysiloxanes), polyethylene glycols, and solid adsorbent- (EU-) fused silica capillary column $(100 \mathrm{~m}$ length, $\quad 0.25 \mathrm{~mm}$ internal diameter $\times 0.2 \mu \mathrm{m}$ film thickness). Nitrogen was used as the carrier gas. The temperature profile was optimized by achieving separated all peaks of interest. Peak retention times were identified by injecting known standards (37 components FAME mix, SUPELCO, USA) with known fatty acid composition. Then, a standard curve was prepared using the 3-point linear plot of different dilutions of the standard, and concentrations $(\mathrm{mg} / \mathrm{ml})$ of individual fatty acids of each sample were calculated.

2.3. Quantitative Real-Time Polymerase Chain Reaction of Apelin Expression. The process of real-time quantitative reverse transcription polymerase chain reaction (qRT-PCR) has previously been reported [5]. Total RNA was isolated from both adipose tissue samples according to manufacturer's protocol of TRIzol reagent (Invitrogen U.S.) and was treated with DNase I to remove traces of genomic DNA. We synthesized cDNA according to manufacturer's protocol by a commercial kit (BioFact, Korea). Real-time PCR (Rotor-Gene 6000 (Sydney, Australia)) was performed by SYBR Green Master Mix (Thermo Scientific, USA). For each gene, samples were run in duplicate for interassay control along with GAPDH and the nontemplate control (NTC). The expression of apelin in each sample was evaluated based on its threshold cycle $(\mathrm{Ct})$, normalized to the $\mathrm{Ct}$ of the reference gene. The minimum information for publication of quantitative real-time PCR experiments (MIQE) were followed [20]. GAPDH was considered as the reference gene for normalization in the samples.

2.4. Covariate Measurements. We assessed habitual dietary intakes of participants using a valid and reliable semiquantitative food frequency questionnaire during an interview by a trained dietitian $[21,22]$. We required participants to elaborate their frequency of consumption daily, weekly, or monthly through the past year. The United States Department of Agriculture food composition table was applied to estimate the nutrients and energy of each food item, except traditional Iranian foods, in which Iranian FCT was used.

Weight and height were measured, and body mass index (BMI) was calculated. Regular physical activity was estimated using the long forms of the reliable and validated Persian version of the International Physical Activity 


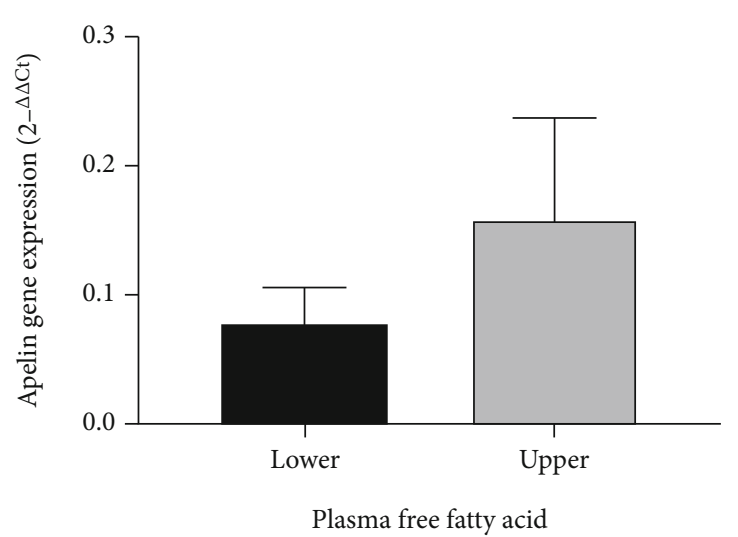

(a)

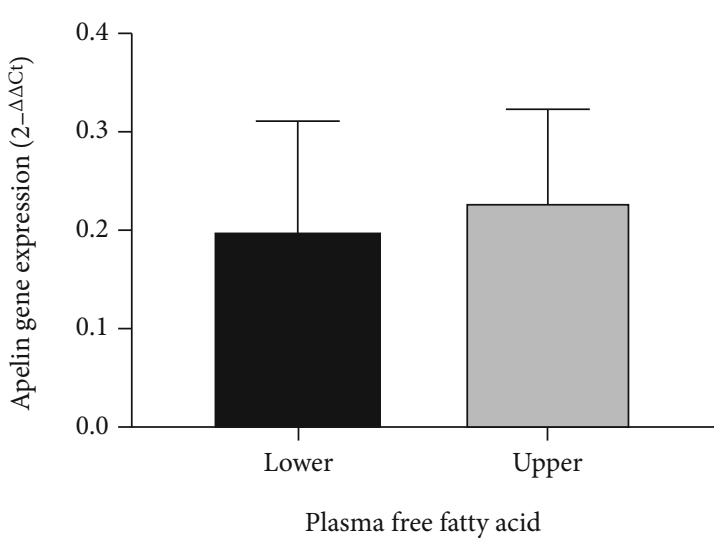

(b)

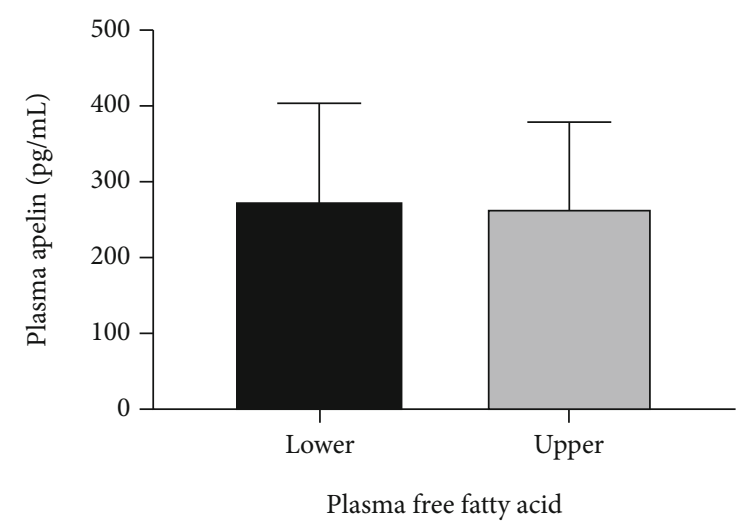

(c)

FIGURE 1: Distribution according to the upper and lower median of plasma free fatty acids regarding (a) apelin gene expression in visceral adipose tissue, (b) apelin gene expression in subcutaneous adipose tissue, and (c) apelin plasma concentration. Results are expressed as mean \pm SD.

Questionnaire through interviews [23]. To measure energy expenditure, the concept of metabolic equivalents (MET) was used. The apelin plasma concentration was measured using the ELISA assay kit (ZellBio, Ulm, Germany), and inter- and intra-assay coefficient of variation (CV) was both $1.9 \%$. Fasting plasma glucose (FPG) was measured using an enzymatic colorimetric method with glucose oxidase (Pars Azmoon Inc., Tehran, Iran). Inter- and intra-assay CVs were both $1.0 \%$ for FPG. Insulin was measured using an enzymelinked immunosorbent assay (ELISA) with Mercodia AB kits (Uppsala, Sweden). Inter- and intra-assay CVs of insulin were $1.7 \%$ and $2.3 \%$, respectively. The homeostatic model assessment of insulin resistance (HOMA-IR) was calculated according to the following formula: [ fasting insulin $(\mu \mathrm{U} / \mathrm{ml}) \times$ fasting glucose $(\mathrm{mmol} / \mathrm{l})] / 22.5$. Participants with HOMA - IR $>3.2$ were considered to be insulin resistant.

2.5. Statistical Analysis. Characteristics of participants were reported by means (standard deviation; SD) and medians $\left(25^{\text {th }}-75^{\text {th }}\right.$ percentile) for normally distributed and skewed continuous variables, respectively, or percentage for categorical variables. The distribution of the variables was checked by the histogram and Kolmogorov-Smirnov tests. Participants were divided based on their median total FFA concen- tration. Differences in biochemical and anthropometric between groups were tested by Student's $t$-test for normally distributed, Wilcoxon rank-sum test for skewed quantitative variables, and by chi-square test for categorical variables.

Multivariable linear regression models were applied to test the associations of total and individual fatty acids (continuous as the independent variable) with apelin gene expression and concentration (continuous as the dependent variable). The model was adjusted for age, sex, BMI, HOMA-IR, physical activity, and energy intake. Analyses were not stratified by sex or BMI because there were no meaningful interactions between total FFAs and these variables in the relationship with apelin gene expression and concentrations when the analysis was run with the inclusion of interaction terms in the regression models. Results from the linear regression models were reported as regression standardized $\beta$ (STZ $\beta$ ), representing unitless variables to show the association of exposures with outcomes. Furthermore, we also reconsidered the goodness of fit and the normality distribution of residuals in the model. All statistical analyses were performed using the statistical package for the social sciences (SPSS) 15.0 software (SPSS Inc., Chicago, IL, USA), and $P$ values $<0.05$ were considered statistically significant. 
TABLE 1: The biochemical and anthropometric characteristics of study participants stratified by median of total plasma free fatty acids.

\begin{tabular}{|c|c|c|c|c|}
\hline & \multirow{2}{*}{ Total } & \multicolumn{2}{|c|}{ Total plasma free fatty acids } & \multirow{2}{*}{$P$ value } \\
\hline & & Lower median & Upper median & \\
\hline Age (years) & $41.1(13.2)$ & $42.9(10.4)$ & $39.2(13.1)$ & 0.069 \\
\hline Female (\%) & 75.0 & 79.5 & 71.5 & 0.164 \\
\hline Body mass index $\left(\mathrm{kg} / \mathrm{m}^{2}\right)$ & $35.6(10.3)$ & $33.7(10.4)$ & $37.6(9.9)$ & 0.011 \\
\hline Fasting plasma glucose (mg/d) & $88.8(18.7)$ & $88.2(19.2)$ & $89.4(18.3)$ & 0.673 \\
\hline Fasting plasma insulin $(\mu \mathrm{U} / \mathrm{ml})$ & $12.3(10.7)$ & $10.6(11.2)$ & $14.0(10.1)$ & 0.034 \\
\hline Insulin resistant (\%) & 31.3 & 23.9 & 38.6 & 0.035 \\
\hline Physical activity (MET/week) & $567(163-1531)$ & $540(179-1793)$ & $585(107-1415)$ & 0.714 \\
\hline \multicolumn{5}{|l|}{ Dietary intake } \\
\hline Total energy intake (kcal) & $2881(1000)$ & $2812(939)$ & $2951(1059)$ & 0.356 \\
\hline Fat (\% energy) & $31.0(6.0)$ & $31.2(6.0)$ & $30.9(6.1)$ & 0.727 \\
\hline Saturated fatty acids (\% energy) & $9.6(2.6)$ & $10.0(2.7)$ & $9.3(2.6)$ & 0.116 \\
\hline Monounsaturated fatty acid (\% energy) & $10.2(2.2)$ & $10.4(2.2)$ & $10.1(2.3)$ & 0.343 \\
\hline Polyunsaturated fatty acid (\% energy) & $6.2(1.7)$ & $6.4(1.6)$ & $6.2(1.7)$ & 0.437 \\
\hline \multicolumn{5}{|l|}{ Plasma fatty acids } \\
\hline Saturated fatty acids & $2.2(1.4)$ & $1.32(0.54)$ & $3.12(1.55)$ & $<0.001$ \\
\hline Lauric acid, C:12 & $0.07(0.07)$ & $0.06(0.06)$ & $0.09(0.07)$ & $<0.001$ \\
\hline Myristic acid, C:14 & $0.32(0.35)$ & $0.20(0.14)$ & $0.45(0.45)$ & $<0.001$ \\
\hline Pentadecanoic acid, C15 & $0.10(0.13)$ & $0.04(0.07)$ & $0.16(0.15)$ & $<0.001$ \\
\hline Palmitic acid, C:16 & $0.86(0.51)$ & $0.56(0.24)$ & $1.17(0.54)$ & $<0.001$ \\
\hline Heptadecanoic acid, C:17 & $0.28(0.51)$ & $0.08(0.13)$ & $0.46(0.66)$ & $<0.001$ \\
\hline Stearic acid, C:18 & $0.50(0.31)$ & $0.33(0.12)$ & $0.68(0.33)$ & $<0.001$ \\
\hline Lignoceric acid, C:24 & $0.07(0.09)$ & $0.04(0.07)$ & $0.10(0.09)$ & $<0.001$ \\
\hline Monounsaturated fatty acid & $1.9(1.9)$ & $0.88(0.42)$ & $2.92(2.27)$ & $<0.001$ \\
\hline Palmitoleic acid, C16:1 & $0.29(0.19)$ & $0.21(0.12)$ & $0.37(0.21)$ & $<0.001$ \\
\hline Oleic acid, C18:1 & $1.42(1.77)$ & $0.36(0.38)$ & $2.21(2.20)$ & $<0.001$ \\
\hline Cis heptadecanoic acid, C17:1 & $0.19(0.57)$ & $0.05(0.15)$ & $0.34(0.77)$ & $<0.001$ \\
\hline Polyunsaturated fatty acid & $4.7(3.90)$ & $2.62(1.02)$ & $6.79(4.52)$ & $<0.001$ \\
\hline Linoleic acid, C18:2 & $3.40(3.66)$ & $1.60(0.87)$ & $5.10(4.40)$ & $<0.001$ \\
\hline Linolenic acid, C18:3 & $0.15(0.09)$ & $0.12(0.08)$ & $0.18(0.09)$ & $<0.001$ \\
\hline Gamma linolenic acid, C18:3 & $0.13(0.09)$ & $0.10(0.08)$ & $0.16(0.08)$ & $<0.001$ \\
\hline Eicosadienoic acid, C20:2 & $0.02(0.04)$ & $0.01(0.03)$ & $0.03(0.05)$ & 0.022 \\
\hline Eicosatetrienoic acid, C20:3 & $0.27(0.15)$ & $0.23(0.11)$ & $0.32(0.16)$ & $<0.001$ \\
\hline Arachidonic acid, C20:4 & $0.45(0.29)$ & $0.35(0.21)$ & $0.10(0.09)$ & $<0.001$ \\
\hline Eicosapentaenoic acid, C20:5 & $0.07(0.09)$ & $0.04(0.07)$ & $0.10(0.09)$ & $<0.001$ \\
\hline Docosahexaenoic acid, C22:6 & $0.20(0.12)$ & $0.16(0.09)$ & $0.24(0.13)$ & $<0.001$ \\
\hline
\end{tabular}

Data are presented as mean (SD) or median (25-75 percentile) for quantitative variables according to their distribution and percent for categorical variables.

\section{Results}

Participants presented a mean age of 41.0 years old, had a mean BMI of $35.6 \mathrm{~kg} / \mathrm{m}^{2}$, and reported a mean total time physical activity energy expenditure of 1822 MET $\times \min$ per week. The fasting plasma apelin concentration ranged from 117 to $561 \mathrm{ng} / \mathrm{ml}$, with a mean of $266 \mathrm{ng} / \mathrm{ml}$. A total of 176 participants were categorized into two groups: low FFAs $(n=88)$ and high FFAs $(n=88)$. There was no significant difference between upper and lower FFAs regarding apelin plasma concentration and gene expression in visceral and subcutaneous adipose tissue (Figure 1).

The biochemical and anthropometric characteristics of study participants are shown in Table 1. We compare two groups of low FFAs and high FFAs using $t$-test and Wilcoxon rank-sum test whenever these were appropriate. The values of BMI were higher in participants with higher FFAs compared to those in the lower FFAs (37.6 (9.9) vs. 33.7 (10.4) $\mathrm{kg} / \mathrm{m}^{2} ; P=0.011$ ). The mean fasting plasma insulin concentration was significantly higher in the high FFAs than in the low FFAs participants (14.0 (10.1) vs. 10.6(11.2) $\mu \mathrm{U} / \mathrm{ml} ; P=0.034)$. As assessed using the HOMA-R formula, the prevalence of insulin resistance was significantly increased in participants with high FFAs compared with low FFAs ones (38.6 vs. $23.9 \% ; P=0.035$ ).

The linear associations of plasma concentration of FFAs with apelin plasma concentrations and gene expression are presented in Table 2. In multivariable analysis, total plasma 
TABle 2: Association of plasma fatty acids with apelin plasma concentration and adipose tissues gene expression.

\begin{tabular}{lcccccc}
\hline & \multicolumn{2}{c}{ Visceral } & \multicolumn{2}{c}{ Subcutaneous } & \multicolumn{2}{c}{ Plasma } \\
& STZ $\beta$ & $P$ value & STZ $\beta$ & $P$ value & STZ $\beta$ & -0.074 \\
Total plasma FFA & 0.241 & 0.006 & 0.040 & 0.655 & 0.389 \\
Saturated fatty acids & 0.336 & $<0.001$ & 0.160 & 0.068 & -0.058 \\
Monounsaturated fatty acid & 0.313 & $<0.001$ & 0.130 & 0.148 & -0.099 \\
Polyunsaturated fatty acid & 0.116 & 0.194 & -0.064 & 0.475 & -0.052 \\
\hline
\end{tabular}

The model was adjusted for age, sex, body mass index, HOMA-IR, physical activity, and energy intake. Multivariable linear regression analyses was performed, and standardized (STZ) $\beta$ were reported.

TABle 3: The associations of plasma concentration of individual fatty acid with apelin plasma concentrations and adipose tissues gene expression.

\begin{tabular}{|c|c|c|c|c|c|c|}
\hline & \multicolumn{2}{|c|}{ Visceral } & \multicolumn{2}{|c|}{ Subcutaneous } & \multicolumn{2}{|c|}{ Plasma } \\
\hline & $\operatorname{STZ} \beta$ & $P$ value & $\operatorname{STZ} \beta$ & $P$ value & $\mathrm{STZ} \beta$ & $P$ value \\
\hline \multicolumn{7}{|l|}{ Saturated fatty acids } \\
\hline Lauric acid, C:12 & 0.068 & 0.383 & -0.070 & 0.370 & 0.066 & 0.361 \\
\hline Myristic acid, C:14 & 0.372 & $<0.001$ & 0.192 & 0.013 & -0.023 & 0.751 \\
\hline Pentadecanoic acid, C15 & 0.252 & 0.002 & 0.186 & 0.023 & 0.052 & 0.499 \\
\hline Palmitic acid, C:16 & 0.035 & 0.659 & -0.050 & 0.523 & -0.055 & 0.448 \\
\hline Heptadecanoic acid, C:17 & 0.335 & $<0.001$ & 0.231 & 0.003 & -0.011 & 0.878 \\
\hline Stearic acid, C:18 & -0.017 & 0.832 & -0.094 & 0.232 & -0.125 & 0.085 \\
\hline Lignoceric acid, C:24 & -0.028 & 0.724 & 0.035 & 0.652 & 0.032 & 0.660 \\
\hline \multicolumn{7}{|l|}{ MUFA } \\
\hline Palmitoleic acid, C16:1 & 0.038 & 0.634 & 0.055 & 0.484 & -0.067 & 0.362 \\
\hline Oleic acid, C18:1 & 0.079 & 0.316 & -0.002 & 0.982 & -0.077 & 0.285 \\
\hline Cis heptadecanoic acid, C17:1 & 0.407 & $<0.001$ & 0.265 & 0.001 & -0.042 & 0.565 \\
\hline \multicolumn{7}{|l|}{ PUFA } \\
\hline Linoleic acid, C18:2 & 0.042 & 0.588 & -0.080 & 0.300 & -0.044 & 0.543 \\
\hline Linolenic acid, C18:3 & 0.156 & 0.046 & 0.077 & 0.322 & 0.008 & 0.910 \\
\hline Gamma linolenic acid, C18:3 & 0.095 & 0.252 & -0.090 & 0.268 & -0.137 & 0.070 \\
\hline Eicosadienoic acid, C20:2 & -0.025 & 0.747 & -0.057 & 0.463 & -0.139 & 0.051 \\
\hline Eicosatetrienoic acid, C20:3 & 0.028 & 0.736 & -0.072 & 0.382 & -0.164 & 0.030 \\
\hline Arachidonic acid, C20:4 & -0.131 & 0.114 & -0.095 & 0.248 & 0.057 & 0.455 \\
\hline Eicosapentaenoic acid, C20:5 & -0.049 & 0.551 & 0.004 & 0.965 & 0.280 & $<0.001$ \\
\hline Docosahexaenoic acid, C22:6 & -0.156 & 0.048 & -0.064 & 0.412 & -0.054 & 0.460 \\
\hline
\end{tabular}

The model was adjusted for age, sex, body mass index, HOMA-IR, physical activity, and energy intake. Multivariable linear regression analyses was performed, and standardized (STZ) $\beta$ were reported.

FFAs (STZ $\beta=0.241, P=0.006)$ were directly associated with apelin gene expression from VAT. Furthermore, SFA $(\mathrm{STZ} \beta=0.336, P<0.001)$ and MUFA $(\mathrm{STZ} \beta=0.313, P<$ $0.001)$ concentration also had a positive association with apelin mRNA levels from VAT after controlling for potential confounders. No significant associations of total FFAs and its subtypes with apelin concentration and SAT mRNA levels were observed.

The associations of plasma concentration of individual fatty acid with apelin plasma concentrations and gene expression are presented in Table 3. After adjusting for potential confounders, the plasma concentration of myristic acid (STZ $\beta=0.372, P<0.001)$, pentadecanoic acid (STZ $\beta=0.252, P=0.002)$, heptadecanoic acid (STZ $\beta=0.335$,
$P<0.001)$, cis-heptadecanoic acid $(\mathrm{STZ} \beta=0.407, \quad P<$ $0.001)$, and linolenic acid (STZ $\beta=0.156, P=0.046)$ was positively associated with apelin mRNA levels in VAT, whereas plasma concertation of DHA was negatively associated with apelin gene expression in VAT. Plasma concentration of myristic acid (STZ $\beta=0.192, P=0.013)$, pentadecanoic acid (STZ $\beta=0.186, P=0.023)$, heptadecanoic acid (STZ $\beta=0.231, P=0.003)$, and cis-heptadecanoic acid (STZ $\beta=0.265, P=0.001$ ) was positively associated with apelin mRNA levels in SAT. In addition, a significant negative association of eicosatetraenoic acid (STZ $\beta=-0.164, P=$ $0.030)$ and a positive association of eicosapentaenoic acid (STZ $\beta=0.280, P<0.001)$ with apelin plasma concentration were observed. 


\section{Discussion}

In the present study of healthy adults, we found that total plasma FFA concentration was associated with the expression of an apelin in VAT after controlling for age, sex, BMI, HOMA-IR, physical activity, and energy intake. There was also a positive association of plasma SFA and MUFA with apelin gene expression from VAT. It should be noted that total FFAs and their subtypes were not associated with apelin concentration as well as SAT mRNA levels.

In recent years, apelin has extensively received attention concerning its role in the progression of insulin resistance. Apelin is expressed and released from adipose tissues, and its regulation is interrupted in obesity and insulin resistance [1]. The stimulatory role of apelin in glucose homeostasis in normal and obese insulin-resistant mice was also proposed [2]. Although there is no study to investigate the association of circulating FFAs with apelin in both serum/plasma concentration and gene expression level, human and animal experiments have demonstrated that apelin concentration and adipose gene expression changed in response to a high-fat diet [24]. Yang et al. illustrated that rats fed with a high-fat diet had higher apelin concentration as well as mRNA expression in adipose tissues [6]. In another study, Bertrand et al. showed that 10 weeks of high-fat diet mice lead to the upregulation of apelin concentration and gene expression in adipose tissue [25]. However, in the current study, we found that higher plasma FFAs were positively associated with apelin gene expression from VAT, unlike the null result observed for apelin concentration. This uncoupled companionship between apelin level in plasma and gene expression was reported in a study with feeding the high-fat diet in rats. After a 7-week intervention by a high-fat diet, the apelin mRNA level in SAT was higher than those in a standard diet, whereas apelin concentration did not change [26]. It seems that apelin gene expression in $\mathrm{VAT}$ is more prone to change in response to the manipulation of fat in a diet than apelin concentration. It is unclear whether adipose tissue contributes markedly to apelin plasma levels.

The results of animal studies support a relation between apelin gene expression and plasma SFAs and MUFAs. One study in rats showed that a diet with a high content of SFA increased apelin gene expression but not apelin plasma concentration [27]. Furthermore, Lasa et al. indicated that apelin concentration and gene expression in hamster feds with sunflower oil, as a source of MUFA, did not differ with standard-fed hamsters [28]. We also observed that myristic acid, pentadecanoic acid, and palmitic acid had a direct association with apelin gene expression in both VAT and SAT in the SFA family. Besides, plasma cis-heptadecanoic acid from the MUFA category had a positive association with apelin gene expression as well. The association of SFAs and MUFAs in the plasma with the transcription of apelin in the VAT suggests the contribution of fatty acid composition along with total FFA concentration in the regulation of apelin in fat depots.

Although studies showed a regulatory effect for PUFA families such as EPA, DHA, linoleic acid, and linolenic acid on the apelin plasma and gene expression in human [29] and animal studies $[25,27]$, we observed a null relationship. The lack of association between PUFAs and their subgroup plasma concentration may pertain to the difference of the various procedures that contributed to the absorption and metabolism PUFA intakes [30-32]. EPA has been reported to have an impact on the gene expression of apelin [33]. Furthermore, in high-fat-fed rats, EPA supplementation increased the apelin mRNA level in VAT [27]. Beyond the exploratory nature of the current study, which revealed association rather than causal linkage, findings warrant further examination using experimental and cohort studies to better understand the impact of plasma FFAs composition on adipokine expression.

Previous studies indicating that the fatty acid composition of plasma mirrors dietary fatty acid consumption and can accordingly be applied as an objective assessment of the type of fatty acids consumed by persons should be noted $[34,35]$. However, since there are difficulties with calculating dietary fat consumption by dietary questionnaire with some inherent measurement error, the application of biomarkers is preferable [36]. For example, in addition to dietary intake, SFAs can be synthesized endogenously via acetyl CoA. Besides, as an abundant member of MUFAs, oleic acid can be produced endogenously through elongation and desaturation of SFAs.

Our study has some limitations. The crosssectional design of the study makes it unclear whether the higher fatty acids in plasma can increase apelin gene expression in adipose tissue. Another limitation of this study was the nonrandom selection of participants, and our sample was recruited from Tehran. Therefore, the results may not be representative of the population. The nature of the nonrandom increases the risk of selection bias and prevents generalization of the findings to the broader population. Despite adjustment for some potential confounders, several other confounders such as genetic background or race may still affect the association between fatty acid concentrations and apelin gene expression. This study also has its strength; it was the first study to provide data on human samples on the association of apelin gene expression and fatty acid concentrations.

\section{Conclusion}

All in all, the current study showed that total plasma FFA concentration was associated with increased VAT apelin gene expression. Furthermore, plasma concentrations SFA and MUFA were associated with higher apelin gene expression from VAT. The direct association of plasma FFA, SFA, and MUFA with apelin gene expression from VAT suggested that plasma fatty acid composition may play an indicatory role in regulating apelin gene expression in VAT.

\section{Abbreviations}

VAT: Visceral adipose tissues

SAT: Subcutaneous adipose tissues

BMI: $\quad$ Body mass index 
$\mathrm{CV}: \quad$ Coefficients of variation

ELISA: Enzyme-linked immunosorbent assay

FPG: $\quad$ Fasting plasma glucose

MET: Metabolic equivalents task

SD: $\quad$ Standard deviation

SPSS: $\quad$ Statistical Package for the Social Sciences program

STZ $\beta$ : Standardized $\beta$

FFAs: $\quad$ Free fatty acids

SFA: $\quad$ Saturated fatty acid

MUFA: Monounsaturated fatty acid

PUFA: Polyunsaturated fatty acid.

\section{Data Availability}

The datasets analyzed during the current study are available from the corresponding author on reasonable request.

\section{Additional Points}

Highlight. A significant positive association of total plasma and circulating plasma FFAs, SFA, and MUFA with apelin gene expression in VAT was observed. There was no association between plasma FA and circulating apelin. It seems that plasma fatty acid composition may regulate apelin gene expression in VAT.

\section{Ethical Approval}

The design of this study was approved by the institutional ethics committee of the Research Institute for Endocrine Sciences, affiliated to the Shahid Beheshti University of Medical Sciences.

\section{Consent}

Written informed consent was obtained from participants.

\section{Disclosure}

The funders had no role in study design, data collection, and analysis, decision to publish, or preparation of the manuscript.

\section{Conflicts of Interest}

The authors declare that they have no competing interests.

\section{Authors' Contributions}

E.Y. conceptualized and designed the study, gathered adipose tissue, performed RNA extraction and real-time PCR, analyzed and interpreted the data, prepared the manuscript, and approved the final manuscript as submitted. G.A. and N.B. entered data, drafted the initial manuscript, and approved the final manuscript as submitted. M.H. supervised the project, consulted lab protocol, and approved the final manuscript as submitted. M.Z. and A.D. prepared the lab materials, cDNA synthesized, performed RNA extraction and real-time PCR, prepared the manuscript, and approved the final manuscript as submitted. P.M. drafted the initial manuscript and approved the final manuscript as submitted.
A.Kh. biopsied the patients during the abdominal surgery and approved the final manuscript as submitted.

\section{Acknowledgments}

We would like to acknowledge Dr. Ebrahimi for his valuable help through the surgery for gathering adipose tissue. This work was funded by the Research Institute for Endocrine Sciences, Shahid Beheshti University of Medical Sciences, Tehran, Iran.

\section{References}

[1] J. Boucher, B. Masri, D. Daviaud et al., "Apelin, a newly identified adipokine up-regulated by insulin and obesity," Endocrinology, vol. 146, no. 4, pp. 1764-1771, 2005.

[2] C. Dray, C. Knauf, D. Daviaud et al., "Apelin stimulates glucose utilization in normal and obese insulin-resistant mice," Cell Metabolism, vol. 8, no. 5, pp. 437-445, 2008.

[3] M. J. Kleinz and A. P. Davenport, "Emerging roles of apelin in biology and medicine," Pharmacology \& Therapeutics, vol. 107, no. 2, pp. 198-211, 2005.

[4] I. Castan-Laurell, R. El Boustany, O. Pereira et al., "Plasma apelin and risk of type 2 diabetes in a cohort from the community," Diabetes Care, vol. 43, no. 2, pp. e15-e16, 2020.

[5] E. Yuzbashian, G. Asghari, M. Aghayan et al., "Dietary glycemic index and dietary glycemic load is associated with apelin gene expression in visceral and subcutaneous adipose tissues of adults," Nutrition and Metabolism, vol. 16, no. 1, 2019.

[6] H. Yang, L. Zhao, J. Zhang, C. S. Tang, Y. F. Qi, and J. Zhang, "Effect of treadmill running on apelin and APJ expression in adipose tissue and skeletal muscle in rats fed a high-fat diet," International Journal of Sports Medicine, vol. 36, no. 7, pp. 535-541, 2015.

[7] K. Tsintzas, K. Chokkalingam, K. Jewell, L. Norton, I. A. Macdonald, and D. Constantin-Teodosiu, "Elevated free fatty acids attenuate the insulin-induced suppression of PDK4 gene expression in human skeletal muscle: potential role of intramuscular long-chain acyl-coenzyme A," The Journal of Clinical Endocrinology \& Metabolism, vol. 92, no. 10, pp. 39673972, 2007.

[8] P. Sbraccia, M. D’Adamo, F. Leonetti et al., "Relationship between plasma free fatty acids and uncoupling protein-3 gene expression in skeletal muscle of obese subjects:in vitroevidence of a causal link," Clinical Endocrinology, vol. 57, no. 2, pp. 199207, 2002.

[9] G. Paolisso, A. Gambardella, L. Amato et al., "Opposite effects of short- and long-term fatty acid infusion on insulin secretion in healthy subjects," Diabetologia, vol. 38, no. 11, pp. 12951299, 1995.

[10] P. Randle, "The glucose fatty-acid cycle its role in insulin sensitivity and the metabolic disturbances of diabetes mellitus," Lancet, vol. 1, no. 7285, pp. 785-789, 1963.

[11] G. M. Reaven, C. Hollenbeck, C.-Y. Jeng, M. S. Wu, and Y.D. I. Chen, "Measurement of plasma glucose, free fatty acid, lactate, and insulin for $24 \mathrm{~h}$ in patients with NIDDM," Diabetes, vol. 37, no. 8, pp. 1020-1024, 1988.

[12] L. H. Opie and P. G. Walfish, "Plasma free fatty acid concentrations in obesity," New England Journal of Medicine, vol. 268 , no. 14 , pp. $757-760,1963$. 
[13] G. Boden and G. Shulman, "Free fatty acids in obesity and type 2 diabetes: defining their role in the development of insulin resistance and $\beta$-cell dysfunction," European Journal of Clinical Investigation, vol. 32, pp. 14-23, 2002.

[14] P. Arner and M. Rydén, "Fatty acids, obesity and insulin resistance," Obesity Facts, vol. 8, no. 2, pp. 147-155, 2015.

[15] S. Santos, A. Oliveira, C. Pinho, S. Casal, and C. Lopes, "Fatty acids derived from a food frequency questionnaire and measured in the erythrocyte membrane in relation to adiponectin and leptin concentrations," European Journal of Clinical Nutrition, vol. 68, no. 5, pp. 555-560, 2014.

[16] C. Enzenbach, J. Kröger, V. Zietemann et al., "Erythrocyte membrane phospholipid polyunsaturated fatty acids are related to plasma C-reactive protein and adiponectin in middle-aged German women and men," European Journal of Nutrition, vol. 50, no. 8, pp. 625-636, 2011.

[17] C. A. Drevon, "Fatty acids and expression of adipokines," Biochimica et Biophysica Acta, vol. 2005, pp. 287-292, 2005.

[18] J. E. Reseland, F. Haugen, K. Hollung et al., "Reduction of leptin gene expression by dietary polyunsaturated fatty acids," Journal of Lipid Research, vol. 42, no. 5, pp. 743-750, 2001.

[19] M. Nosrati-Oskouie, E. Yuzbashian, M. Zarkesh et al., "Association of plasma fatty acids pattern with omentin gene expression in human adipose tissues: a cross-sectional study," Nutrition, Metabolism, and Cardiovascular Diseases, vol. 31, no. 3, pp. 894-901, 2021.

[20] S. A. Bustin, V. Benes, J. A. Garson et al., "The MIQE guidelines: minimum information for publication of quantitative real-time PCR experiments," Clinical Chemistry, vol. 55, no. 4, pp. 611-622, 2009.

[21] G. Asghari, A. Rezazadeh, F. Hosseini-Esfahani, Y. Mehrabi, P. Mirmiran, and F. Azizi, "Reliability, comparative validity and stability of dietary patterns derived from an FFQ in the Tehran lipid and glucose study," The British Journal of Nutrition, vol. 108, no. 6, pp. 1109-1117, 2012.

[22] P. Mirmiran, F. H. Esfahani, Y. Mehrabi, M. Hedayati, and F. Azizi, "Reliability and relative validity of an FFQ for nutrients in the Tehran lipid and glucose study," Public Health Nutrition, vol. 13, no. 5, pp. 654-662, 2010.

[23] A. Vasheghani-Farahani, M. Tahmasbi, H. Asheri, H. Ashraf, S. Nedjat, and R. Kordi, "The Persian, last 7-day, long form of the International Physical Activity Questionnaire: translation and validation study," Asian Journal of Sports Medicine, vol. 2, p. 106, 2011.

[24] E. Yuzbashian, M. Zarkesh, G. Asghari et al., "Is apelin gene expression and concentration affected by dietary intakes? A systematic review," Critical Reviews in Food Science and Nutrition, vol. 58, no. 4, pp. 680-688, 2018.

[25] C. Bertrand, A. Pignalosa, E. Wanecq et al., "Effects of dietary eicosapentaenoic acid (EPA) supplementation in highfat fed mice on lipid metabolism and apelin/APJ system in skeletal muscle," PLoS One, vol. 8, no. 11, article e78874, 2013.

[26] D. F. Garcia-Diaz, J. Campion, A. V. Arellano, F. I. Milagro, M. J. Moreno-Aliaga, and J. A. Martinez, "Fat intake leads to differential response of rat adipocytes to glucose, insulin and ascorbic acid," Experimental Biology and Medicine, vol. 237, no. 4, pp. 407-416, 2012.

[27] N. Pérez-Echarri, P. Pérez-Matute, B. Marcos-Gómez, J. A. Martínez, and M. J. Moreno-Aliaga, "Effects of eicosapentaenoic acid ethyl ester on visfatin and apelin in lean and over- weight (cafeteria diet-fed) rats," The British Journal of Nutrition, vol. 101, no. 7, pp. 1059-1067, 2009.

[28] A. Lasa, I. Churruca, E. Simón et al., "Effects of high-fat highsucrose feeding, energy restriction, and trans-10,cis-12 conjugated linoleic acid on visfatin and apelin in hamsters," Journal of the American College of Nutrition, vol. 28, no. 6, pp. 627635, 2009.

[29] A. Mortazavi, E. Nematipoor, M. Djalali et al., "The effect of omega-3 fatty acids on serum apelin levels in cardiovascular disease: a randomized, double-blind, placebo-controlled trial," Reports of biochemistry \& molecular biology, vol. 7, no. 1, pp. 59-66, 2018.

[30] F. H. Chilton, R. C. Murphy, B. A. Wilson et al., "Diet-gene interactions and PUFA metabolism: a potential contributor to health disparities and human diseases," Nutrients, vol. 6, no. 5, pp. 1993-2022, 2014.

[31] D. Lelli, R. Antonelli Incalzi, L. Ferrucci, S. Bandinelli, and C. Pedone, "Association between PUFA intake and serum concentration and mortality in older adults: a cohort study," Clinical Nutrition, vol. 39, no. 2, pp. 510-515, 2020.

[32] T. Y. Wang, M. Liu, P. Portincasa, and D. Q. H. Wang, "New insights into the molecular mechanism of intestinal fatty acid absorption," European Journal of Clinical Investigation, vol. 43, no. 11, pp. 1203-1223, 2013.

[33] S. Lorente-Cebrián, M. Bustos, A. Marti, J. A. Martinez, and M. J. Moreno-Aliaga, "Eicosapentaenoic acid up-regulates apelin secretion and gene expression in 3T3-L1 adipocytes," Molecular Nutrition \& Food Research, vol. 54, Suppl 1, pp. S104-S111, 2010.

[34] S. K. Raatz, D. Bibus, W. Thomas, and P. Kris-Etherton, "Total fat intake modifies plasma fatty acid composition in humans," The Journal of Nutrition, vol. 131, no. 2, pp. 231-234, 2001.

[35] J. Ma, A. R. Folsom, E. Shahar, and J. H. Eckfeldt, "Plasma fatty acid composition as an indicator of habitual dietary fat intake in middle-aged adults. The Atherosclerosis Risk in Communities (ARIC) study investigators," The American Journal of Clinical Nutrition, vol. 62, no. 3, pp. 564-571, 1995.

[36] J. K. Bassett, A. M. Hodge, D. R. English, R. J. MacInnis, and G. G. Giles, "Plasma phospholipids fatty acids, dietary fatty acids, and breast cancer risk," Cancer Causes \& Control, vol. 27, no. 6, pp. 759-773, 2016. 